\title{
A lower bound for the area of Plateau foams
}

Vicent Gimeno ${ }^{1 *}$, Steen Markvorsen ${ }^{2}$ and José M. Sotoca ${ }^{3}$

"Correspondence: gimenov@uji.es 'Departament de

Matemàtiques-IMAC, Universitat Jaume I, Castelló, Spain

Full list of author information is available at the end of the article

\begin{abstract}
Real foams can be viewed as geometrically well-organized dispersions of more or less spherical bubbles in a liquid. When the foam is so drained that the liquid content significantly decreases, the bubbles become polyhedral-like and the foam can be viewed now as a network of thin liquid films intersecting each other at the Plateau borders according to the celebrated Plateau's laws.

In this paper we estimate from below the surface area of a spherically bounded piece of a foam. Our main tool is a new version of the divergence theorem which is adapted to the specific geometry of a foam with special attention to its classical Plateau singularities.

As a benchmark application of our results, we obtain lower bounds for the fundamental cell of a Kelvin foam, lower bounds for the so-called cost function, and for the difference of the pressures appearing in minimal periodic foams. Moreover, we provide an algorithm whose input is a set of isolated points in space and whose output is the best lower bound estimate for the area of a foam that contains the given set as its vertex set.
\end{abstract}

Keywords: Foams; Bubbles; Density; Pressure; Comparison geometry

\section{Introduction}

A foam is a cell decomposition of the Euclidean 3 -space $\mathbb{R}^{3}$ into a finite or infinite number of properly embedded, connected 3D chambers. Here, the chambers are not assumed to be either compact or homeomorphic to a ball in $\mathbb{R}^{3}$, but to qualify as a foam we will assume that they comply with the famous Plateau rules.

The Plateau rules are the following: Firstly, the interfaces between neighboring chambers all have constant mean curvature; secondly, the interfaces meet in threes (at equal $2 \pi / 3$ angles) along smooth edges; and thirdly, the edges always meet four at a time in isolated points, where the angle between any pair of edges is precisely $\arccos (-1 / 3)$.

A foam is clearly represented by its $0-, 1-$, and 2 -skeleton, i.e., the union of its faces, edges, and vertices. We will typically denote this union by $F$. The constant mean curvature of the surface of each face in a foam is proportional to the pressure difference between the two cells meeting along the face. Every foam is organized around two angles: Every vertex treats the foam like the center vertex that locates the 6 inner wings in a regular tetrahedron, and every edge organizes three of these wings to have equal angles $2 \pi / 3$ between them, see Fig. 1.

(c) The Author(s) 2020. This article is licensed under a Creative Commons Attribution 4.0 International License, which permits use sharing, adaptation, distribution and reproduction in any medium or format, as long as you give appropriate credit to the original author(s) and the source, provide a link to the Creative Commons licence, and indicate if changes were made. The images or other third party material in this article are included in the article's Creative Commons licence, unless indicated otherwise in a credit line to the material. If material is not included in the article's Creative Commons licence and your intended use is not permitted by statutory regulation or exceeds the permitted use, you will need to obtain permission directly from the copyright holder. To view a copy of this licence, visit http://creativecommons.org/licenses/by/4.0/. 

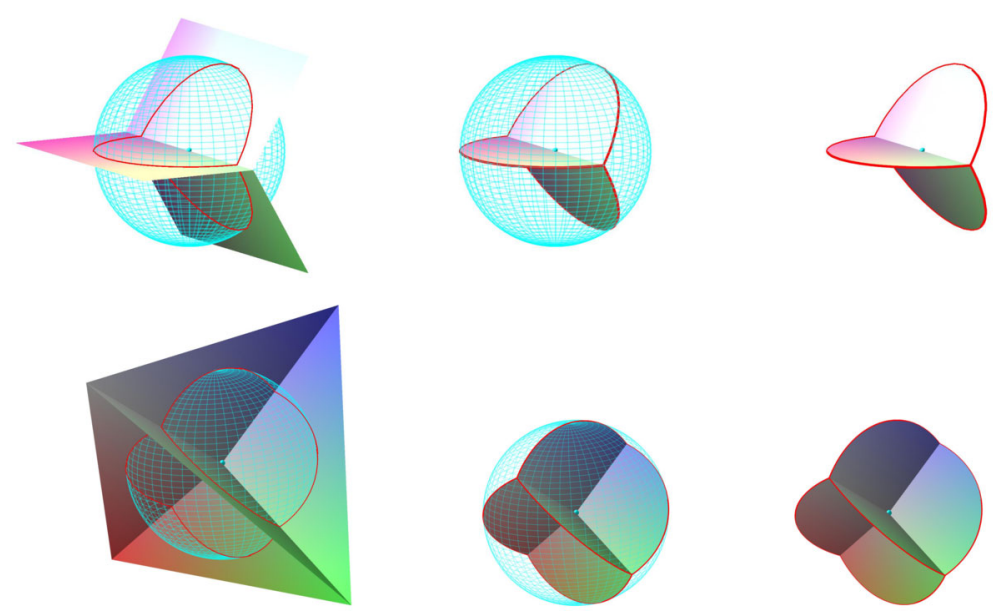

Figure 1 Cutting off spherical scoops_extrinsic foam discs_-from affine foams around an edge point (top row) and a vertex point (bottom row), respectively

Plateau found these rules in the nineteenth century when he was studying the geometry of assembled bubbles in equilibrium. From then on, foams have been largely studied because of their amazing physical and mathematical properties related, e.g., to packings and isoperimetric problems, see $[7,15,19]$.

The goal of this paper is to obtain lower bounds for the area of 'spherical scoops' of a foam. Locally the area of the tetrahedral linear foam (totally geodesic) inside a sufficiently small ball of radius $R$ centered at a foam vertex is similar to $\pi \theta_{v} R^{2}$, where $\theta_{v}=$ $\frac{3}{\pi} \arccos (-1 / 3)$ is the density of the tetrahedral 6-wing construction at the vertex, see Fig. 1 . This is the estimated area that we will apply for comparison at every vertex of the given foam in order to achieve an effective lower bound for the total area of a foam.

We will assume throughout that the length of the mean curvature vector of the faces of the foam $F$ is bounded by a constant $h$ as follows:

$$
|H(x)| \leq h \quad \text { for all } x \in F
$$

Our main objective is to determine a lower bound for the area of an extrinsic disc (a 'spherical scoop') of a foam centered at a given vertex point. An extrinsic disc of radius $R$ centered at a vertex point is the intersection of a ball of the Euclidean 3-space of radius $R$ centered at the vertex point of the foam. In Fig. 2 we show a numerical simulation of an extrinsic disc of the Kelvin foam obtained by using the Surface Evolver program. In general, an extrinsic disc does not have to be centered at a vertex point. Indeed, in this paper we define it as follows.

Definition 1.1 (Extrinsic disc) Let $F \subset \mathbb{R}^{3}$ be a foam. An extrinsic disc $D_{R}(o)$ of radius $R$ centered at $o$ is the intersection $F \cap B_{R}(o)$, where $B_{R}(o)$ denotes the open Euclidean distance ball with radius $R$ and center $o$ in $\mathbb{R}^{3}$.

\subsection{Main result. Area comparison}

Let $F$ be a foam satisfying the mean curvature bound (1). The main result of this paper states that, for any point $o$ (which is not necessarily a vertex point) in $F$ and for every 

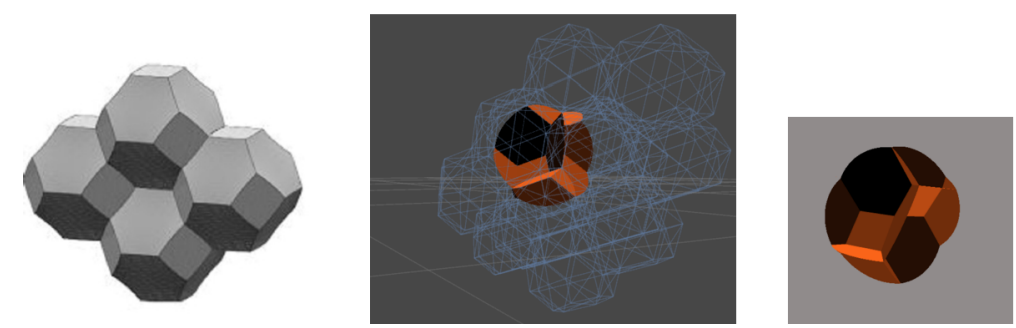

Figure 2 Piece of the Kelvin foam and an (enlarged) extrinsic disc of the Kelvin foam centered at a vertex point

spherical scoop extrinsic foam disc $D_{R}(o)$ of radius $R$ centered at $o$, a sharp lower bound for the area of the extrinsic disc can be obtained in terms of the radius, the upper bound for the norm of the mean curvature vector field, and the type of the point $o$.

More precisely, we can state the following main theorem.

Main Theorem Let $F$ be a foam properly immersed satisfying the mean curvature bound (1). Then the area of the extrinsic foam disc $D_{R}(o)$ of radius $R$ centered at o is bounded from below by

$$
A\left(D_{R}(o)\right) \geq \theta(o) \cdot e^{-2 h R} \cdot \pi R^{2},
$$

where

$$
\theta(o)= \begin{cases}\theta_{v}=\frac{3}{\pi} \arccos (-1 / 3) & \text { if o is a vertex of } F \\ \theta_{e}=\frac{3}{2} & \text { if o lies in an edge of } F \\ \theta_{f}=1 & \text { if o lies in a face of } F\end{cases}
$$

Furthermore, equality in inequality (2) is attained if and only if every face element of the extrinsic foam disc $D_{R}(o)$ is a piece of an affine plane containing $o$.

Remark 1.1 This main theorem can be viewed as a concrete explication of a general result of [1] with special focus on the particular structural data that appear for Plateau foam varifolds in $\mathbb{R}^{3}$. In this paper we provide a proof using an adapted version of the divergence theorem for foams. Moreover, in Theorem 5.2 we extend the main theorem and obtain an even sharper global lower area bound for foams using a specific optimal measure on the vertex set of the respective foams, see Definition 5.1.

Simplest examples 1.1 The simplest non-planar example of a foam consists of just one spherical bubble $F$ with 1 face (of radius $\rho$ ), no edges and no vertices. See Fig. 3. In this case, Theorem 1.1 says that, for any point $o$ on the sphere, the area of the extrinsic $R$-disc centered at $o$ is

$$
\mathrm{A}\left(F \cap B_{R}(o)\right) \geq \pi R^{2} \cdot e^{-2 R / \rho},
$$



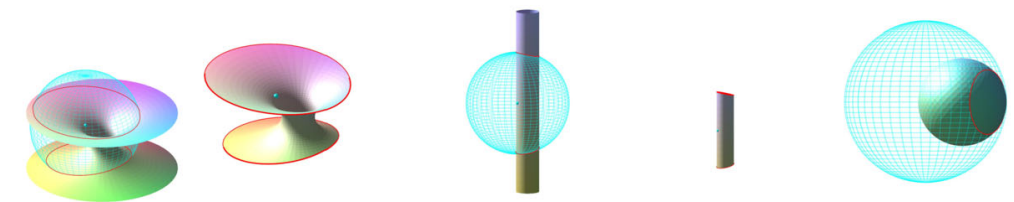

Figure 3 From left to right: cutting off extrinsic foam discs from a catenoid, a cylinder, and a sphere

where we have used the optimal mean curvature bound $h=1 / \rho$ for $F$. Inequality (4) is easily seen to be true for all $R$ independent of the given $\rho$, since in this case we have

$$
\mathrm{A}\left(F \cap B_{R}(o)\right)= \begin{cases}\pi R^{2} & \text { for } R \leq 2 \rho \\ 4 \pi \rho^{2} & \text { for } R \geq 2 \rho .\end{cases}
$$

Another simple example $F$ with 1 face, no edges, and no vertices is the cylinder with radius $\rho$, which is also on display in Fig. 3. The estimate of Theorem 1.1 is the same as before:

$$
\mathrm{A}\left(F \cap B_{R}(o)\right) \geq \pi R^{2} \cdot e^{-2 R / \rho} .
$$

Again this is easily verified; in particular, for very large $R \gg \rho$, the area is approximately given by

$$
\mathrm{A}\left(F \cap B_{R}(o)\right) \approx 2 \pi R \cdot \rho \gg \pi R^{2} e^{-2 R / \rho} .
$$

More precisely, for any finite value of $R$, the extrinsic $R$-disk contains the intrinsic flat $R$ disk in the cylinder (with the overlapping segments of the flat intrinsic disk removed in case $R>\pi \rho$ ), so that the following estimate holds for all $R$ and $\rho$ :

$$
\mathrm{A}\left(F \cap B_{R}(o)\right)> \begin{cases}\pi R^{2} & \text { for } R \leq \pi \rho, \\ \pi R^{2}-2\left(R^{2} \arccos \left(\frac{\pi \rho}{R}\right)-\pi \rho \sqrt{R^{2}-(\pi \rho)^{2}}\right) & \text { for } R \geq \pi \rho .\end{cases}
$$

It follows directly that $\mathrm{A}\left(F \cap B_{R}(o)\right) \geq \pi R^{2} e^{-2 R / \rho}$ for all $R$ and $\rho$ as claimed.

Finally, we should mention also the example supplied by the catenoid $F$. This is a minimal surface, so $h=0$, and for any given point $o \in F$ and very large $R$ the intersection $F \cap B_{R}(o)$ is essentially two large discs of radius $R$ as indicated on the left in Fig. 3. In consequence, the area estimate is thus

$$
\mathrm{A}\left(F \cap B_{R}(o)\right) \approx 2 \pi R^{2}>\pi R^{2}
$$

which again gives a rough verification of the theorem. We also mention here that the comparison $\mathrm{A}\left(F \cap B_{R}(o)\right) \geq \pi R^{2}$ has in fact previously been proved for all values of $R$ (and for general minimal submanifolds in bounded ambient curvatures) via heat kernel comparison methods (see, e.g., [3, 13], and [14]).

As already alluded to, the main tool of the present paper is the statement and proof of an adapted version of the divergence theorem for foams, Theorem 3.1. 


\section{Outline of the paper}

In Sect. 3 we introduce all required propositions and preliminaries, including the adapted version of the divergence theorem, needed to prove the main theorem of the paper in Sect. 4. The last part of the paper, Sect. 5, is devoted to showing some applications of the main theorem. These applications are as follows:

1 A lower bound for the area of a compact foam, Sect. 5.1.

2 A recipe on how to use the main theorem to obtain a lower bound for the contribution of the area of a cell to the total area of a foam. Actually, in Sect. 5.2, we show how to obtain, via the main theorem, a lower bound for the area of a cell in the Kelvin foam.

3 A lower bound for the cost function in Sect. 5.3 and a lower bound for the pressure in minimal foams in Sect. 5.4.

4. Finally, in Sect. 5.5, we provide an algorithm to compute a lower bound for the area of a foam using only the positions of the vertices of the foam.

\section{Preliminaries}

By using an appropriate version of the divergence theorem on extrinsic discs of foams and the general co-area formula given by geometric measure theory, we state the main comparison theorem for the area of extrinsic foam discs.

In a foam $F \subset \mathbb{R}^{3}$ there are three different kinds of points: the points on the interior of the faces, the points on the interior of the edges, and the points on the vertices. Each face in a foam is a smooth CMC surface (i.e., with constant norm of the mean curvature vector field). They are orientable and support individually a well-defined normal vector field. We can consider that each face of the foam is embedded in $\mathbb{R}^{3}$.

\subsection{Intrinsic and extrinsic distance function on a foam}

Given the inclusion map $i: F \rightarrow \mathbb{R}^{3}$ for the foam to the Euclidean space $\mathbb{R}^{3}$, we will say that a map $\gamma: I \rightarrow F$ from the interval $I \subset \mathbb{R}$ is a piecewise smooth curve segment of $F$ if the composed map with the inclusion $\tilde{\gamma}: I \rightarrow \mathbb{R}^{3}, \tilde{\gamma}=i \circ \gamma$ is a piecewise smooth curve segment of $\mathbb{R}^{3}$. Every piecewise smooth curve segment $\gamma$ has length $\mathrm{L}_{F}(\gamma)$ given by the length of the associated piecewise smooth curve segment $\widetilde{\gamma}$ in $\mathbb{R}^{3}$, i.e., $\mathrm{L}_{F}(\gamma)=\mathrm{L}(\widetilde{\gamma})$.

Given two points, $p, q \in F$ we can now define the intrinsic distance from $p$ to $q$, denoted by $\operatorname{dist}_{F}(p, q)$, to be the infimum of $\mathrm{L}(\gamma)$ over all piecewise smooth curve segments $\gamma$ from $p$ to $q$. With this definition, $\left(F\right.$, dist $\left._{F}\right)$ becomes a metric space.

If we choose a point $o \in \mathbb{R}^{3}$, we can define the extrinsic distance function $r_{o}: F \rightarrow \mathbb{R}$ by

$$
r_{o}(x)=\operatorname{dist}_{\mathbb{R}^{3}}(o, x) .
$$

Hence, for any two points $p, q \in F$,

$$
\left|r_{o}(p)-r_{o}(q)\right| \leq \operatorname{dist}_{\mathbb{R}^{3}}(p, q) \leq \operatorname{dist}_{F}(p, q) .
$$

That means that the extrinsic distance function is a 1-Lipschitz function on the metric space $\left(F\right.$, dist $\left._{F}\right)$. Observe that, since each face $\left\{F_{i}\right\}$ of $F$ is a smooth surface of $\mathbb{R}^{3}$, then the extrinsic distance function is a $C^{\infty}$ function on $F_{i} \backslash\{o\}$. Moreover, for any $v_{i} \in T_{p} F_{i}$,

$$
d r_{o}\left(v_{i}\right)=\left\langle\nabla^{F} r_{o}, v_{i}\right\rangle=\left\langle\nabla^{\mathbb{R}^{3}} r_{o}, v_{i}\right\rangle \leq\left\|v_{i}\right\|
$$


which implies

$$
\left\|\nabla^{F_{i}} r_{o}\right\| \leq 1
$$

By using Sard's theorem, the set of critical values of $r_{o}: F_{i} \backslash\{o\} \rightarrow \mathbb{R}_{+}$has zero Lebesgue measure in $\mathbb{R}^{+}$. The set of sublevel sets of the extrinsic distance is precisely the extrinsic disc, namely

$$
D_{R}(o)=\left\{x \in F: r_{o}(x)<R\right\}=F \cap B_{R}(o) .
$$

The boundary of the extrinsic disc $D_{R}$ will be denoted by $\partial D_{R}$, i.e.,

$$
\partial D_{R}(o)=\left\{x \in F: r_{o}(x)=R\right\}=F \cap S_{R}(o) .
$$

Similarly, for $\rho<R$, the extrinsic annulus is

$$
A_{\rho, R}(o):=\left\{x \in F: \rho \leq r_{o}(x) \leq R\right\} .
$$

Hence, by using the co-area formula (see [18] for instance), we can state the following.

Proposition 3.1 Let $D_{R}(o)$ be an extrinsic disc of a foam $F$. Suppose that $R$ is a regular value of $r_{o}: F_{i} \backslash\{o\} \rightarrow \mathbb{R}$ for every face $F_{i}$ of $F$, and suppose, moreover, that $\partial D_{R}$ meets transversally every edge of $F$. Then

$$
\left.\frac{d}{d t} \mathrm{~A}\left(D_{t}(o)\right)\right|_{t=R}=\sum_{i} \int_{F_{i} \cap \partial D_{R}} \frac{d L_{i}}{\left\|\nabla^{i} r_{o}\right\|}
$$

\subsection{Divergence theorem on foams}

Let us now, without loss of generality, center a point $o \in F$ of the foam $F$ in the origin of $\mathbb{R}^{3}$ and consider the extrinsic disc $D_{R}(0)=F \cap B_{R}(0)$ of radius $R$ and center $0 \in \mathbb{R}^{3}$. We impose, moreover, that $R$ is such that every edge meets $\partial D_{R}$ transversally. Such an extrinsic disc $D_{R}(0)$ centered at $0 \in \mathbb{R}^{3}$ is not a smooth surface but is composed of a finite number $f_{R}$ of faces (which are in fact individually smooth surfaces), a finite number of edges $e_{R}$, and a finite number of vertices $v_{R}$. The faces, edges, and vertices of $D_{R}(0)$ are intersections of faces, edges, and vertices of $F$ with the ball $B_{R}(0)$. Hence,

$$
D_{R}(0)=\left\{F_{i}\right\}_{i=1}^{f_{R}} \cup\left\{E_{i}\right\}_{i=1}^{e_{R}} \cup\left\{V_{i}\right\}_{i=1}^{v_{R}},
$$

where $\left\{F_{i}\right\}_{i=1}^{f_{R}},\left\{E_{i}\right\}_{i=1}^{e_{R}},\left\{V_{i}\right\}_{i=1}^{v_{R}}$ are the sets of faces, edges, and vertices respectively. Let us denote

$$
\begin{aligned}
& \mathcal{F}_{R}:=\left\{F_{i}\right\}_{i=1}^{f_{R}}, \\
& \mathcal{E}_{R}:=\left\{E_{i}\right\}_{i=1}^{e_{R}}, \\
& \mathcal{V}_{R}:=\left\{V_{i}\right\}_{i=1}^{v_{R}} .
\end{aligned}
$$


Let us denote by $n_{i}$ a unit normal vector field to the face $F_{i}$. Hence, given a smooth vector field $X: B_{R}(0) \rightarrow T \mathbb{R}^{3}$ in $B_{R}(0) \subset \mathbb{R}^{3}$, we can obtain a vector field $X_{F_{i}}$ tangent and smooth on each face $F_{i}$ by using the normal vector field $n_{i}$ in such a way that

$$
X_{F_{i}}:=X-\left\langle X, n_{i}\right\rangle n_{i}
$$

Denote by $X_{F}$ the application $X_{F}: F \backslash \mathcal{E}_{R} \rightarrow T \mathbb{R}^{3}$ given by

$$
X_{F}(x)=X_{F_{i}}(x) .
$$

On every face $F_{i}$ of $F$, if $R$ is a regular value of $r_{o}$, the vector field $\nabla^{F_{i}} r_{o}=\nabla^{\mathbb{R}^{3}} r_{o}-$ $\left\langle\nabla^{\mathbb{R}^{3}} r_{o}, n_{i}\right\rangle n_{i}$ never vanishes on $F_{i} \cap \partial B_{R}(0)$, in fact,

$$
v_{i}^{R}=\frac{\nabla^{F_{i}} r_{o}}{\left\|\nabla^{F_{i}} r_{o}\right\|}=\frac{\nabla^{\mathbb{R}^{3}} r_{o}-\left\langle\nabla^{\mathbb{R}^{3}} r_{o}, n_{i}\right\rangle n_{i}}{\left\|\nabla^{\mathbb{R}^{3}} r_{o}-\left\langle\nabla^{\mathbb{R}^{3}} r_{o}, n_{i}\right\rangle n_{i}\right\|}
$$

is the outward unit normal vector field to $\partial D_{R}$ on $F_{i} \cap \partial B_{R}(0)$.

With these definitions we can state the following divergence theorem on foams.

Theorem 3.1 (Divergence theorem on foams) Let $F \subset \mathbb{R}^{3}$ be a foam in $\mathbb{R}^{3}$. Let $D_{R}(0)$ be the extrinsic disc given by $D_{R}(0)=F \cap B_{R}(0)$, then for any vector field $X: B_{R} \rightarrow T \mathbb{R}^{3}$

$$
\int_{D_{R}(0)} \operatorname{div} X_{F} d A=\int_{\partial D_{R}(0)}\left\langle X, v_{F}\right\rangle d L
$$

where

$$
\int_{\partial D_{R}(0)}\left\langle X, v_{F}\right\rangle d L:=\sum_{i=1}^{f_{R}} \int_{F_{i} \cap \partial B_{R}(0)}\left\langle X, v_{i}^{R}\right\rangle d L_{i}
$$

Proof We can directly relate the integral of the divergence of the tangential component $X_{F}$ of vector field $X \in \mathfrak{X}\left(\mathbb{R}^{3}\right)$ to the sum of the divergence in each face. Namely,

$$
\int_{D_{R}(0)} \operatorname{div} X_{F} d A:=\sum_{i=1}^{f_{R}} \int_{F_{i}} \operatorname{div} X_{F_{i}} d A_{i} .
$$

Observe that on each face $F_{i}$ we can have two kinds of boundary components of $\partial F_{i}$ :

$$
\partial F_{i}=\left(F_{i} \cap \partial B_{R}(0)\right) \cup\left(F_{i} \cap \mathcal{E}_{R}\right) .
$$

Accordingly, let us denote by $v_{i}^{R}$ the outward unit normal vector field on $F_{i} \cap \partial B_{R}(0)$ with $v_{i}^{R}=0$ if $F_{i} \cap \partial B_{R}(0)=\emptyset$ and by $v_{i, j}$ the outward unit normal vector field on $F_{i} \cap e_{j}$ with $v_{i, j}=0$ if $F_{i} \cap e_{j}=\emptyset$. Observe that by the structure of the foam and the three-faces-oneedge with equal angles we have

$$
\sum_{i=1}^{f_{R}} v_{i, j}=0
$$


for any $j \in\left\{1, \ldots, e_{R}\right\}$. Hence, by using the divergence theorem on each face (see Theorem 14.34 of [12] for instance), we get

$$
\begin{aligned}
\int_{D_{R}(0)} \operatorname{div} X_{F} d A & =\sum_{i=1}^{f_{R}} \int_{F_{i}} \operatorname{div} X_{F_{i}} d A_{i} \\
& =\sum_{i=1}^{f_{R}}\left(\int_{F_{i} \cap \partial B_{R}(0)}\left\langle X_{F_{i}}, v_{i}^{R}\right\rangle d L_{i}+\sum_{j=1}^{e_{R}} \int_{F_{i} \cap \varrho_{j}}\left\langle X_{F_{i}}, v_{i, j}\right\rangle d L_{i, j}\right) \\
& =\sum_{i=1}^{f_{R}}\left(\int_{F_{i} \cap \partial B_{R}(0)}\left\langle X, v_{i}^{R}\right\rangle d L_{i}+\sum_{j=1}^{e_{R}} \int_{e_{j}}\left\langle X, v_{i, j}\right\rangle d L_{j}\right) \\
& =\sum_{i=1}^{f_{R}} \int_{F_{i} \cap \partial B_{R}(0)}\left\langle X, v_{i}^{R}\right\rangle d L_{i}+\sum_{j=1}^{e_{R}} \int_{e_{j}}\left\langle X, \sum_{i=1}^{f_{R}} v_{i, j}\right\rangle d L_{j} \\
& =\sum_{i=1}^{f_{R}} \int_{F_{i} \cap \partial B_{R}(0)}\left\langle X, v_{i}^{R}\right\rangle d L_{i} .
\end{aligned}
$$

\subsection{Laplacian of the coordinate functions of $\mathbb{R}^{3}$ on a CMC surface}

Recall that given a smooth surface $S \subset \mathbb{R}^{3}$ in the Euclidean space $\mathbb{R}^{3}$, the covariant derivatives on $S$ and $\mathbb{R}^{3}$ are related by the Gauss formula by

$$
\nabla_{\bar{X}}^{\mathbb{R}^{3}} \bar{Y}=\nabla_{X}^{S} Y+\alpha(X, Y)
$$

for any two vector fields $X, Y \in \mathfrak{X}(S)$ and any two extensions $\bar{X}, \bar{Y} \in \mathfrak{X}\left(\mathbb{R}^{3}\right)$. The term $\alpha$ is the second fundamental form of $S$. Similarly, the Hessian operators Hess ${ }^{S}$ and Hess $\mathbb{R}^{3}$ are related by the following.

Proposition 3.2 Let $f: \mathbb{R}^{3} \rightarrow \mathbb{R}$ be a smooth function, and let us denote also by $f: S \rightarrow \mathbb{R}$ the restriction of the function to the smooth surface $S \subset \mathbb{R}^{3}$, then

$$
\operatorname{Hess}^{S} f(X, Y)=\operatorname{Hess}^{\mathbb{R}^{3}} f(\bar{X}, \bar{Y})+\left\langle\alpha(X, Y), \nabla^{\mathbb{R}^{3}} f\right\rangle
$$

for any two vector fields $X, Y \in \mathfrak{X}(S)$ and any two extensions $\bar{X}, \bar{Y} \in \mathfrak{X}\left(\mathbb{R}^{3}\right)$.

Proof

$$
\begin{aligned}
\operatorname{Hess}^{S} f(X, Y) & =\left\langle\nabla_{X}^{S} \nabla^{S} f, Y\right\rangle=X\left(\left\langle\nabla^{S} f, Y\right\rangle\right)-\left\langle\nabla^{S} f, \nabla_{X}^{S} Y\right\rangle \\
& =X\left(\left\langle\nabla^{\mathbb{R}^{3}} f, Y\right\rangle\right)-\left\langle\nabla^{\mathbb{R}^{3}} f, \nabla_{X}^{S} Y\right\rangle \\
& =X\left(\left\langle\nabla^{\mathbb{R}^{3}} f, Y\right\rangle\right)-\left\langle\nabla^{\mathbb{R}^{3}} f, \nabla_{X}^{\mathbb{R}^{3}} Y\right\rangle+\left\langle\nabla^{\mathbb{R}^{3}} f, \alpha(X, Y)\right\rangle \\
& =\operatorname{Hess}^{\mathbb{R}^{3}} f(\bar{X}, \bar{Y})+\left\langle\alpha(X, Y), \nabla^{\mathbb{R}^{3}} f\right\rangle .
\end{aligned}
$$

The above proposition leads us to the following one. 
Proposition 3.3 Let $\varphi: S \rightarrow \mathbb{R}^{3}$ be an immersed surface in $\mathbb{R}^{3}$. Let us denote by $\{x, y, z\}$ the coordinate functions in $\mathbb{R}^{3}$ and their restrictions to $S$. Let

$$
H=\frac{1}{2} \operatorname{trace}_{g}(\alpha)=\left(H_{1}, H_{2}, H_{3}\right)
$$

be the mean curvature vector field of S. Then

$$
\begin{aligned}
& \triangle^{S} x=2 H_{1}, \\
& \triangle^{S} y=2 H_{2}, \\
& \triangle^{S} z=2 H_{3} .
\end{aligned}
$$

Proof Let us consider the following vector $a=\left(a_{1}, a_{2}, a_{3}\right)$ and the following function:

$$
f_{a}: \mathbb{R}^{3} \rightarrow \mathbb{R}, \quad(x, y, z) \rightarrow f_{a}(x, y, z)=a_{1} x+a_{2} y+a_{3} z
$$

One can easily check that

$$
\nabla^{\mathbb{R}^{3}} f_{a}=a,
$$

and hence

$$
\operatorname{Hess}^{\mathbb{R}^{3}} f_{a}(\bar{X}, \bar{Y})=0, \quad \forall \bar{X}, \bar{Y} \in \mathfrak{X}\left(\mathbb{R}^{3}\right) .
$$

Using therefore Proposition 3.2,

$$
\operatorname{Hess}^{S} f_{a}(X, Y)=\left\langle\nabla^{\mathbb{R}^{3}} f_{a}, \alpha(X, Y)\right\rangle=\langle a, \alpha(X, Y)\rangle,
$$

thus, for any orthonormal basis $\left\{e_{1}, e_{2}\right\}$ of $T_{p} S$ at $p \in S$,

$$
\triangle^{S} f_{a}=\sum_{i=1}^{2} \operatorname{Hess}^{S} f_{a}\left(e_{i}, e_{i}\right)=\sum_{i=1}^{2}\left\langle a, \alpha\left(e_{i}, e_{i}\right)\right\rangle=\left\langle a, \sum_{i=1}^{2} \alpha\left(e_{i}, e_{i}\right)\right\rangle=2\langle a, H\rangle .
$$

Finally, the proposition follows for the particular cases $a=(1,0,0)$ or $a=(0,1,0)$ or $a=$ $(0,0,1)$.

\subsection{Density of a foam}

We denote by $\theta(o)$ the density of the point $o$, i.e., (see also Definition 2.5 of [15]),

$$
\theta(o)=\lim _{s \rightarrow 0^{+}} \frac{\mathrm{A}\left(D_{s}(o)\right)}{\pi s^{2}} .
$$

Since we are assuming that the foam is embedded, the density of a point in a face is just 1 because the area of the extrinsic disk $D_{s}(o)$ of the face is equal to the area of the extrinsic disk of the corresponding flat tangent plane $T_{o} F$ up to and including Taylor order 2 in $s$ around $s=0^{+}$(see, e.g., [8] for further elaborations in this vein). The extrinsic disk of radius $s$ centered at an edge point, or at a vertex point, consists of 3 , respectively 6 , identical sectors (wedge areas) of a full disk with wedge angles $\pi$ and $\arccos (-1 / 3)$, respectively. Since a sector of angle $\phi$ has area $s^{2} \phi / 2$ (up to Taylor order 2 in $s$ ), we then get the following. 
Proposition 3.4 Let $F \subset \mathbb{R}^{3}$ be a foam. Then, for any $o \in F$,

$$
\theta(o)= \begin{cases}\frac{3}{\pi} \arccos (-1 / 3) & \text { ifo is a vertex of } F \\ \frac{3}{2} & \text { ifo lies in the interior of an edge of } F, \\ 1 & \text { ifo lies in the interior of a face of } F\end{cases}
$$

\section{Proof of the main theorem}

In Proposition 4.2 we will be able to obtain a lower bound for the area of an extrinsic disc of foam. First we need the following.

Proposition 4.1 Let $D_{R}(o)$ be an extrinsic disc of a foam $F$. Suppose that $R$ is a regular value of $r_{o}: F_{i} \backslash\{o\} \rightarrow \mathbb{R}$ for every face $F_{i}$ of $F$, and suppose, moreover, that $\partial D_{R}$ meets transversally every edge of $F$. Then

$$
\mathrm{A}\left(D_{R}\right)(1-h R) \leq \frac{R}{2} \sum_{i=1}^{f_{R}} \int_{F_{i} \cap \partial D_{R}}\left|\nabla^{F_{i}} r_{o}\right| d L_{i} .
$$

Proof Without loss of generality suppose that $o=0 \in \mathbb{R}^{3}$. We are using the divergence theorem on foams (Theorem 3.1) with the vector field in $B_{R}(o)$ given by

$$
X=\nabla^{\mathbb{R}^{3}} \phi
$$

where $\nabla^{\mathbb{R}^{3}}$ is the gradient of $\mathbb{R}^{3}$ and

$$
\phi=\frac{1}{4}\left(x^{2}+y^{2}+z^{2}\right) .
$$

$x, y, z$ being the coordinate functions in $\mathbb{R}^{3}$. Hence, on every face $F_{i} \subset F$

$$
\begin{aligned}
X_{F_{i}} & =\nabla^{\mathbb{R}^{3}} \phi-\left\langle\nabla^{\mathbb{R}^{3}} \phi, n_{i}\right) n_{i}=\nabla^{F_{i}} \phi_{\mid F_{i}} \\
& =\frac{1}{2}\left(x \nabla^{F_{i}} x+y \nabla^{F_{i}} y+z \nabla^{F_{i}} z\right) .
\end{aligned}
$$

Thus,

$$
\begin{aligned}
\operatorname{div} X_{F_{i}}= & \frac{1}{2}\left(\left\langle\nabla^{F_{i}} x, \nabla^{F_{i}} x\right\rangle+\left\langle\nabla^{F_{i}} y, \nabla^{F_{i}} y\right\rangle+\left\langle\nabla^{F_{i}} z, \nabla^{F_{i}} z\right\rangle\right) \\
& +\frac{1}{2}\left(x \triangle^{F_{i}} x+y \triangle^{F_{i}} y+z \triangle^{F_{i}} z\right)
\end{aligned}
$$

Denoting by $H$ the mean curvature of the face $F_{i}$ and applying Proposition 3.3, we have

$$
\begin{aligned}
\operatorname{div} X_{F_{i}}= & \frac{1}{2}\left(\left|\nabla^{F_{i}} x\right|^{2}+\left|\nabla^{F_{i}} y\right|^{2}+\left|\nabla^{F_{i}} z\right|^{2}\right)+\langle\vec{r}, H\rangle \\
= & \frac{1}{2}\left(\left|\nabla^{\mathbb{R}^{3}} x\right|^{2}-\left\langle\nabla^{\mathbb{R}^{3}} x,\left.n\right|^{2}+\left|\nabla^{\mathbb{R}^{3}} y\right|^{2}\right.\right. \\
& -\left\langle\nabla^{\mathbb{R}^{3}} y,\left.n\right|^{2}+\left|\nabla^{\mathbb{R}^{3}} z\right|^{2}-\left\langle\nabla^{\mathbb{R}^{3}} z,\left.n\right|^{2}\right)+\langle\vec{r}, H\rangle\right. \\
= & \frac{1}{2}\left(3-|n|^{2}\right)+\langle\vec{r}, H\rangle=1+\langle\vec{r}, H\rangle .
\end{aligned}
$$


Here, $\vec{r}=(x, y, z)$. Then

$$
\operatorname{div} X_{F_{i}} \geq 1-|H| R \geq 1-h R \text {. }
$$

Using Theorem 3.1 and denoting $\partial D_{R}^{i}=F_{i} \cap \partial D_{R}$, we obtain

$$
\begin{aligned}
\mathrm{A}\left(D_{R}(o)\right)(1-h R) & \leq \sum_{i=1}^{f_{R}} \int_{F_{i}} \operatorname{div} X_{F_{i}} d A_{i}=\sum_{i=1}^{f_{R}} \int_{\partial D_{R}^{i}}\left\langle X, v_{i}^{R}\right\rangle d L_{i} \\
& =\sum_{i=1}^{f_{R}} \int_{\partial D_{R}^{i}}\left\langle\nabla^{\mathbb{R}^{3}} \phi, v_{i}^{R}\right\rangle d L_{i}=\sum_{i=1}^{f_{R}} \int_{\partial D_{R}^{i}} \frac{r_{o}}{2}\left\|\nabla^{F_{i}} r_{o}\right\| d L_{i} .
\end{aligned}
$$

Proposition 4.2 Let $F$ be a foam. For any $R>0$, denote by $h$ the maximum of the norm of the mean curvature vector field in the faces of $D_{R}$, i.e.,

$$
h=\max _{x \in D_{R}}\|\vec{H}(x)\|
$$

Then, for any $\rho>0$ such that $\rho<R$,

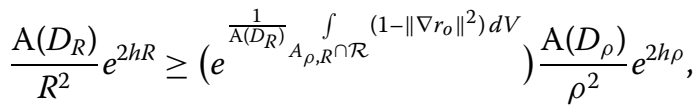

where $\nabla$ is the intrinsic gradient.

Proof Let us denote

$$
\mathcal{R}:=\left\{\begin{array}{c}
x \in F \mid r_{o}(x) \text { is a regular value and } \\
\partial D_{r_{0}(x)} \text { meets the edges of } F \text { transversally }
\end{array}\right\}
$$

By using co-area formula, Proposition 3.1, and Proposition 4.1, we get

$$
\begin{aligned}
\frac{1}{\mathrm{~A}\left(D_{R}\right)} \int_{A_{\rho, R} \cap \mathcal{R}}\left(1-\left\|\nabla r_{o}\right\|^{2}\right) d V & =\frac{1}{\mathrm{~A}\left(D_{R}\right)} \int_{\rho}^{R}\left(\int_{\partial D_{t}} \frac{1-\left\|\nabla r_{o}\right\|^{2}}{\left\|\nabla r_{o}\right\|} d \mathrm{~A}_{t}\right) d t \\
& \leq \int_{\rho}^{R} \frac{1}{\mathrm{~A}\left(D_{t}\right)}\left(\int_{\partial D_{t}}\left(\frac{1}{\left\|\nabla r_{o}\right\|}-\left\|\nabla r_{o}\right\|\right) d \mathrm{~A}_{t}\right) d t \\
& \leq \int_{\rho}^{R} \frac{1}{\mathrm{~A}\left(D_{t}\right)}\left(\frac{d}{d t} \mathrm{~A}\left(D_{t}\right)-\frac{2}{t} \mathrm{~A}\left(D_{t}\right)(1-h t)\right) d t \\
& =\int_{\rho}^{R}\left(\frac{d}{d t} \log \left(\mathrm{A}\left(D_{t}\right)\right)-\frac{d}{d t}\left(\log \left(t^{2}\right)-2 h t\right)\right) d t
\end{aligned}
$$

Taking into account that the function $t \rightarrow \mathrm{A}\left(D_{t}\right)$ is $C^{\infty}$ almost everywhere in $[\rho, R]$ and nondecreasing, then

$$
\log \left(\frac{\frac{\mathrm{A}\left(D_{R}\right)}{R^{2}} e^{2 h R}}{\frac{\mathrm{A}\left(D_{\rho}\right)}{\rho^{2}} e^{2 h \rho}}\right) \geq \frac{1}{\mathrm{~A}\left(D_{R}\right)} \int_{A_{\rho, R} \cap \mathcal{R}}\left(1-\left\|\nabla r_{o}\right\|^{2}\right) d V \geq 0 .
$$

Hence the proposition follows. 
By using the above proposition,

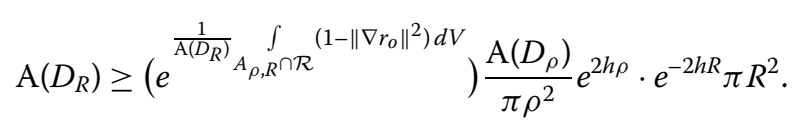

Thence, letting $\rho$ tend to 0 and using Proposition 3.4, we obtain

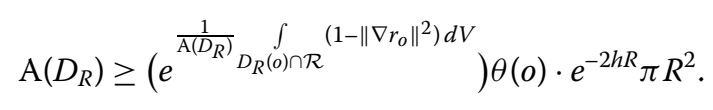

This inequality leads us to the statement and proof of the main theorem.

Main Theorem Let $F$ be a foam properly immersed satisfying the mean curvature bound (1). Then the area of the extrinsic disc $D_{R}(o)$ of radius $R$ centered at o is bounded from below by

$$
A\left(D_{R}(o)\right) \geq \theta(o) \cdot e^{-2 h R} \cdot \pi R^{2},
$$

where

$$
\theta(o)= \begin{cases}\theta_{v}=\frac{3}{\pi} \arccos (-1 / 3) & \text { if o is a vertex of } F \\ \theta_{e}=\frac{3}{2} & \text { if o lies in the interior of an edge of } F, \\ \theta_{f}=1 & \text { if o lies in the interior of a face of } F .\end{cases}
$$

Furthermore, the equality in inequality (47) is attained if and only if every face of the extrinsic foam disc $D_{R}(o)$ is a piece of an affine plane containing $o$.

Proof Observe that inequality (47) follows from inequality (46). Moreover, the equality in inequality (47) implies the equality in (46), therefore

$$
\left\|\nabla r_{o}\right\|^{2}=1
$$

for every point in $D_{R}(o) \cap \mathcal{R}$. Then every face of the extrinsic disc $D_{R}(o)$ is a piece of an affine plane containing $o$.

\section{Applications}

\subsection{Compact foams}

We apply our results to the setting of compact foams.

Theorem 5.1 Let $F \subset \mathbb{R}^{3}$ be a compact foam. Then, given a point $o \in F$, there exists $R_{\max }<$ $\infty$ such that $F=D_{R_{\max }}(o)$, there exists $h<\infty$ such that $\|\vec{H}\|(x) \leq h$ for any $x \in F$. The maximum radius $R_{\max }$ and the supremum of the norm of the mean curvature vector field are related by

$$
R_{\max } \geq \frac{1}{h}
$$

Moreover, the foam has finite area $\mathrm{A}(F)<\infty$ and is bounded from below by

$$
\mathrm{A}(F) \geq \frac{\theta(o)}{e^{2}} \frac{\pi}{h^{2}} .
$$


Proof By Proposition 4.1 we know that

$$
\mathrm{A}\left(D_{R}\right)(1-h R) \leq \mathrm{L}\left(\partial D_{R}\right)
$$

where $\mathrm{L}\left(\partial D_{R}\right)$ denotes the length of the boundary $\partial D_{R}$. Let now $\left\{R_{i}\right\}$ be a sequence for regular values of the extrinsic distance converging to $R_{\max }$, then

$$
\mathrm{A}\left(D_{R_{\max }}\right)\left(1-h R_{\max }\right)=\lim _{i \rightarrow \infty} \mathrm{A}\left(D_{R_{i}}\right)\left(1-h R_{i}\right) \leq \lim _{i \rightarrow \infty} \mathrm{L}\left(\partial D_{R_{i}}\right)=0 .
$$

Hence we obtain

$$
R_{\max } \geq \frac{1}{h}
$$

Finally, by using the main theorem, we have

$$
\mathrm{A}(F) \geq \mathrm{A}\left(D_{R_{\max }}\right) \geq \mathrm{A}\left(D_{1 / h}\right) \geq \frac{\theta(o)}{e^{2}} \frac{\pi}{h^{2}} .
$$

Simplest examples 5.1 The simplest example of a compact foam is a sphere $S_{R}$ of radius $R$ in $\mathbb{R}^{3}$. From any base point on the sphere, we have $R_{\max }=2 R$ so that

$$
R_{\max }=2 R=\frac{2}{h} \geq \frac{1}{h}
$$

which is in complete agreement with the inequality $R_{\max } \geq \frac{1}{h}$. On the other hand, since

$$
\mathrm{A}\left(S_{R}\right)=4 \pi R^{2}=\frac{4 \pi}{h^{2}} \geq \frac{\theta(o)}{e^{2}} \frac{\pi}{h^{2}}=\frac{\pi}{e^{2} h^{2}} .
$$

Another simple example of a compact foam is a double bubble (see Fig. 4). The maximum of the mean curvature is

$$
h=\frac{1}{r_{2}} .
$$

Hence, by the above corollary,

$$
R_{\max } \geq r_{2}
$$

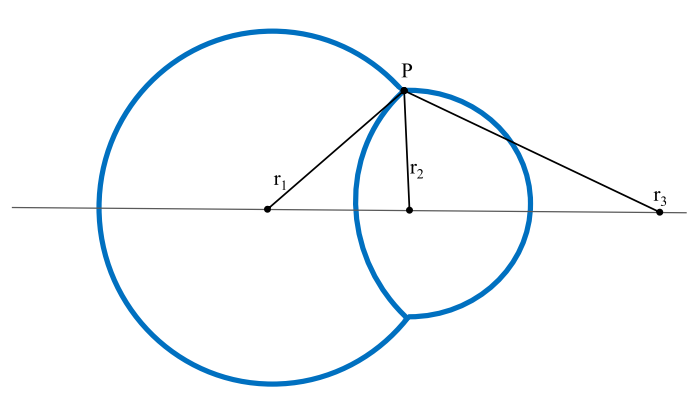

Figure 4 Double bubble foam 
If we choose the center in the point $P$, it is easy to check that $R_{\max }=2 r_{1} \geq r_{2}$. The lower bound for the area given by the above corollary is

$$
A(F) \geq \frac{3}{2 e^{2}} \pi r_{2}^{2} .
$$

\subsection{Lower bound for the area of a cell of a Kelvin foam}

The accuracy of the lower bounds for the area of a domain of a foam given by any method based on the main theorem relies drastically on the number and the distribution of vertex (or face, or edge) points that are chosen. If we choose very few points, the inequalities given by the main theorem are far away from being sharp, but we can nevertheless obtain a lower bound. In this subsection, as an example of an application, we show how to use the main theorem directly to obtain a lower bound for the area of a foam. In this case we use a cell of the Kelvin foam (where lower bounds for the area function are known).

The Kelvin foam arises from the problem of partitioning space into equal-volume cells, using the least interface area. Kelvin described his foam as a relaxation of the Voronoi partition for the body-centered cubic lattice, whose cells are congruent truncated octahedra. As the foam relaxes, see [11], the symmetry present in the Voronoi partition is preserved, and hence the vertices are fixed by symmetry. The square faces remain in their mirror planes, although the edges bend within these planes. The diagonals of the hexagons remain fixed along axes of rotational symmetry, while the hexagons become shaped like monkey-saddles (see [11] and Fig. 2). Observe that the resulting foam is a minimal foam, i.e., it has $h=0$.

In this subsection we are interested in a lower bound for the contribution of each cell to the total area of the foam. Observe that each face contributes in half part of its area to the total area of the foam (since each face is in contact with two cells). We consider an extrinsic disc centered at the center of each hexagonal face (these centers remain fixed under the foam relaxation). If we denote by $a$ the (extrinsic) distance between two adjacent vertices, and if we choose the radii of such extrinsic discs as $R_{1}=\frac{a}{2}$ (less than $\frac{3}{4} a$, the maximal distance between the center of the hexagons and the planes where the square faces lie) and apply the main theorem, then we obtain the following lower bound for the area of these extrinsic foam discs:

$$
A_{1}={ }^{\text {number of hexagonal faces }} 8 \frac{1}{2} \cdot \pi\left(\frac{a}{2}\right)^{2} .
$$

Now we can put an extrinsic disc of radius $R_{2}=\frac{a}{2}$ in each vertex, but now these discs contribute only the fourth part to the total area because identical cells meet at four vertices, and this gives us the following lower bound:

$$
A_{2}=\stackrel{\text { number of vertices }}{24} \cdot \frac{1}{4} \cdot 3 \arccos (-1 / 3)\left(\frac{a}{2}\right)^{2} \text {. }
$$

Since the square faces remain in their mirror planes but with edges bent within these planes, the original square (of area $a^{2}$ ) is contained in the final relaxed square face. Then we obtain the lower bound

$$
A_{3}=\underset{\text { number of square faces }}{6} \cdot \frac{1}{2}\left(a^{2}-\pi\left(\frac{a}{2}\right)^{2}\right)
$$


where we have subtracted the contribution of the discs of the four vertices. Finally, we obtain

Area of a cell of the Kelvin foam $\geq A_{1}+A_{2}+A_{3} \approx 12.3832 a^{2}$.

Observe that by using a "slicing argument" the best lower bound estimation is obtained in $[11]$ as

$$
\text { Area of a cell of the Kelvin foam }>6\left(\sqrt{\frac{3}{2}}+1\right) a^{2} \approx 13.3485 a^{2} .
$$

\subsection{Lower bounds for the cost function}

A classical mathematical problem arising from the honeycomb conjecture and the Kelvin problem is how the space $\mathbb{R}^{3}$ could be partitioned into disjoint cells of equal volume with the least area of surface between them. When we try to compare two different partitions of the space, we need to apply a precise scale invariant quantity. One of the most used quantities is the cost function (see [11])

$$
\mu=\frac{\mathrm{A}^{3}}{\mathrm{~V}^{2}},
$$

where $\mathrm{A}$ is the average interface area per cell and $\mathrm{V}$ is the average cell volume.

Given a foam $F \subset \mathbb{R}^{3}$ and a domain $\Omega \subset \mathbb{R}^{3}$ such that $F \cap \Omega \neq \emptyset$ (with perhaps $\Omega=\mathbb{R}^{3}$ ), studying the scale-invariant cost function, we get-from an isoperimetric point of viewan estimate for how effective the foam fills the domain $\Omega$. Here

$$
\mu(\Omega):=\frac{(\mathrm{A}(F \cap \Omega) / n)^{3}}{(\mathrm{~V}(\Omega) / n)^{2}}
$$

where $n$ denotes the number of cells inside $\Omega$. In the particular case of a minimal foam we can state a lower bound for this cost function reading off only information about the vertices and their distribution.

Given such a domain $\Omega$, let us use the following notation:

$$
\begin{aligned}
& n:=\text { number of cells inside } \Omega, \\
& v_{\Omega}:=\text { number of vertices inside } \Omega, \\
& \bar{v}_{\Omega}:=\frac{v_{\Omega}}{n}, \\
& d:=\text { minimal extrinsic distance between vertices, } \\
& v_{\Omega}:=\frac{v_{\Omega}}{\mathrm{V}(\Omega)} .
\end{aligned}
$$

Corollary 5.1 Let $F \subset \mathbb{R}^{3}$ be a minimal foam in $\mathbb{R}^{3}$, hence for any domain $\Omega \subset \mathbb{R}^{3}$,

$$
\mu(\Omega) \geq \bar{v}_{\Omega} v_{\Omega}^{2} A_{0}^{3}(d)
$$

where $A_{0}$ is given by

$$
A_{0}(d)=\theta_{v} \pi\left(\frac{d}{2}\right)^{2}, \quad \theta_{\nu}=\frac{3}{\pi} \arccos \left(-\frac{1}{3}\right) .
$$


Proof By using the main theorem,

$$
\mu(\Omega)=\frac{1}{n} \frac{(\mathrm{A}(F \cap \Omega))^{3}}{(\mathrm{~V}(\Omega))^{2}} \geq \frac{1}{n} \frac{\left(v_{\Omega} \theta_{v} \pi d^{2}\right)^{3}}{(\mathrm{~V}(\Omega))^{2}}=\left(\frac{v_{\Omega}}{n}\right)\left(\frac{v_{\Omega}}{\mathrm{V}(\Omega)}\right)^{2}\left(\theta_{v} \pi\left(\frac{d}{2}\right)^{2}\right)^{3} .
$$

A classical related problem arising from Kelvin's problem is concerned with the study of the optimization of the cost function under partitions of the space in periodic domains (see [11]). A partition of space is a division of $\mathbb{R}^{3}$ into disjoint cells. We are mainly interested in the surfaces forming the interface between cells. The partitions that we consider will be periodic with respect to some lattice, with some number $n$ of cells in each periodic domain. Obviously, we can use periodic minimal foams as examples of such partitions of space.

In such a case, by using the lower bound given in [10] for the average number of vertices in a periodic minimal foam, we get

$$
\bar{v}_{\Omega} \geq 24 \text {. }
$$

Corollary 5.2 Let $F \subset \mathbb{R}^{3}$ be a minimal and periodic foam in $\mathbb{R}^{3}$, hence for any domain $\Omega \subset \mathbb{R}^{3}$,

$$
\mu(\Omega) \geq 24 v_{\Omega}^{2} A_{0}^{3}(d)
$$

\subsection{Lower bound for the pressure on minimal foams}

A problem which is related to the isoperimetric properties is concerned with the pressure distribution in a foam. We consider a dried foam in mechanical equilibrium with an ideal gas in the bubble chambers. The gas in each chamber is assumed to satisfy the ideal gas equation of state, and we assume that the entire foam is held at constant temperature. In such a case the ratio of area per volume is fixed by the physical constraints involving the temperature, pressure, and surface tension. Indeed, if we have a minimal foam $F \subset \Omega$ inside a region $\Omega \subset \mathbb{R}^{3}$, since the foam is minimal, each cell is at the same pressure $p_{\text {in }}$, but there is also an extrinsic pressure $p_{\text {ext }}$ coming from the boundary $\partial \Omega$, and those two pressures are related as follows (see $[2,4-6,17])$ :

$$
p_{\text {int }}-p_{\text {ext }}=\frac{3}{2} \sigma \frac{\mathrm{A}(F)}{\mathrm{V}(\Omega)}
$$

where $\sigma$ is the surface tension assumed to be homogeneous throughout the foam. Hence,

$$
p_{\text {int }}-p_{\text {ext }}=\frac{3}{2} \sigma\left(\frac{n}{\mathrm{~V}(\Omega)} \mu(\Omega)\right)^{\frac{1}{3}} .
$$

The pressure inside of each cell in the foam can therefore be estimated from below using only the vertices as

$$
p_{\text {int }} \geq p_{\text {ext }}+\frac{3}{2} \sigma v_{\Omega} A_{0}(d) .
$$




\subsection{Area and extrinsic vertex area}

Given a domain $\Omega \in \mathbb{R}^{3}$, the main result allows us to construct an algorithm to estimate lower bounds for the area of the domain $\Omega \cap F \subset F$ of a foam $F$. Given a finite set of points $X=\left\{x_{1}, x_{2}, \ldots, x_{N}\right\} \subset \Omega$. This set $X$ has associated the following subset of $\mathbb{R}^{N}$ :

$$
\begin{aligned}
D_{\Omega}(X):= & \left\{\left(r_{1}, r_{2}, \ldots, r_{N}\right) \subset \mathbb{R}^{N} \mid B_{r_{i}}\left(x_{i}\right) \subset \Omega \text { for } i=1, \ldots, N\right. \\
& \text { and } \left.B_{r_{i}}\left(x_{i}\right) \cap B_{r_{j}}\left(x_{j}\right)=\emptyset \text { for all } i \neq j\right\} .
\end{aligned}
$$

We define the extrinsic vertex area of $X$ in $\Omega$ as follows.

Definition 5.1 Given a set of isolated points $X$ in $\Omega \subset \mathbb{R}^{3}$, the lower extrinsic vertex area of $X$ with $h$ weight is given by

$$
\operatorname{eva}(X, \Omega, h):=\max _{\left(r_{1}, \ldots, r_{N}\right) \in D_{\Omega}(X)}\left\{\sum_{i} e^{-2 h r_{i}} \theta_{v} \cdot \pi r_{i}^{2}\right\}
$$

where $D_{\Omega}(X)$ is given by Definition (61) and $\theta_{v}=\frac{3}{\pi} \arccos (-1 / 3)$. The extrinsic vertex area of $X$ with $h$ weight is given by

$$
\operatorname{evA}(X, \Omega, h):=\max _{Z \subset X}\{\operatorname{eva}(Z, \Omega, h)\} .
$$

Observe that the extrinsic vertex area associates an area to a set of points. By using our main theorem, we can make use of the extrinsic vertex area associated to a set of points $X \subset \Omega$ to obtain a lower bound for the area of a piece of the foam $\Omega \cap F \subset F$.

Theorem 5.2 Let $F \subset \mathbb{R}^{3}$ be a foam, and let $\Omega \subset \mathbb{R}^{3}$ and assume that $\Omega \cap F$ is a compact subset of $F$. Denote by $\mathcal{V}_{\Omega}$ the set of vertices of $\Omega \cap F$, and denote by

$$
h=\max _{x \in \Omega \cap F}\|\vec{H}\|(x)
$$

the maximum of the norm of the mean curvature vector field on the faces of $\Omega \cap F$. Then the area of $\Omega \cap F$ is bounded from below by

$$
A(\Omega \cap F) \geq \operatorname{evA}\left(X, \mathcal{V}_{\Omega}, h\right)
$$

where $\operatorname{evA}\left(X, \mathcal{V}_{\Omega}, h\right)$ is the extrinsic vertex area of the vertex points $\mathcal{V}_{\Omega}$ with $h$ weight.

Remark 5.1 Observe, moreover, that since by definition

$$
\operatorname{evA}(X, \Omega, h) \geq \operatorname{eva}(X, \Omega, h)
$$

then under the hypothesis of the above theorem we can state

$$
\mathrm{A}(\Omega \cap F) \geq \operatorname{eva}\left(X, \mathcal{V}_{\Omega}, h\right) .
$$

Remark 5.2 Given a set of $N$ isolated points $X$ in $\Omega \subset \mathbb{R}^{3}$, the corresponding $D_{\Omega}(X)$ can be understood as the set of admissible radii in order to place the extrinsic foam discs to estimate the largest lower bound for the area of such a set of extrinsic discs. But observe 
that since the function

$$
f: \mathbb{R} \rightarrow \mathbb{R}, \quad r \rightarrow f(r)=e^{-2 h r} r^{2}
$$

is increasing only in the interval $\left[0, \frac{1}{h}\right)$ (with $[0, \infty)$ if $h=0$ ), then we can define the maximal radius $r_{\max }:=\frac{1}{h}$ and the maximal hypercube $B_{r_{\max }}^{\infty}:=\left[0, r_{\max }\right]^{N}$ in such a way that

$$
\operatorname{eva}(X, \Omega, h)=\max _{\left(r_{1}, \ldots, r_{N}\right) \in D_{\Omega}(X) \cap B_{r_{\max }}^{\infty}}\left\{\sum_{i} e^{-2 h r_{i}} \theta_{v} \cdot \pi r_{i}^{2}\right\} .
$$

Stated otherwise, the maximum in Definition 5.1 is obtained in

$$
\left(r_{1}, \ldots, r_{N}\right) \in D_{\Omega}(X) \quad \text { with } r_{i} \leq r_{\max }=\frac{1}{h} \text { for } i=1, \ldots, N .
$$

The presence of mean curvature on the faces of a foam forces, therefore, our method to make use only of small extrinsic discs.

\subsubsection{From the matrix of distances to the extrinsic vertex area}

Let now $X=\left\{x_{1}, \ldots, x_{N}\right\}$ be a set of $N$ isolated points inside a compact subset $\Omega \subset \mathbb{R}^{3}$ with boundary $\partial \Omega$. We can define the matrix of distances $\mathcal{D}_{\Omega}(X)$ of $X$ in $\Omega$ to be the $(N+1) \times(N+1)$ matrix given by

$$
\left[\mathcal{D}_{\Omega}(X)\right]_{i, j}:= \begin{cases}\operatorname{dist}_{\mathbb{R}^{3}}\left(x_{i}, x_{j}\right) & \text { if } i \leq N \text { and } j \leq N \\ \operatorname{dist}_{\mathbb{R}^{3}}\left(x_{i}, \partial \Omega\right) & \text { if } i \leq N \text { and } j=N+1 \\ \operatorname{dist}_{\mathbb{R}^{3}}\left(x_{j}, \partial \Omega\right) & \text { if } i=N+1 \text { and } j \leq N \\ 0 & \text { if } i=N+1 \text { and } j=N+1\end{cases}
$$

From this matrix we can define as well the reduced matrix of distances to be the $N \times N$ matrix given by

$$
\left[d_{\Omega}(X)\right]_{i, j}:=\min \left\{\left[\mathcal{D}_{\Omega}(X)\right]_{i, j},\left[\mathcal{D}_{\Omega}(X)\right]_{i, N+1},\left[\mathcal{D}_{\Omega}(X)\right]_{j, N+1}, \frac{1}{h}\right\}
$$

if $N>1$, or by

$$
\left[d_{\Omega}(X)\right]_{1,1}:=\min \left\{\operatorname{dist}_{\mathbb{R}^{3}}\left(x_{1}, \partial \Omega\right), \frac{1}{h}\right\}
$$

if $N=1$. Observe that

$$
D_{\Omega}(X) \cap B_{r_{\max }}^{\infty}=\left\{r \in \mathbb{R}_{+}^{N} \mid 0 \leq r_{i}+r_{j} \leq\left[d_{\Omega}(X)\right]_{i, j}, \forall i \text { and } \forall j \neq i\right\}
$$

if $N>1$, and

$$
D_{\Omega}(X) \cap B_{r_{\max }}^{\infty}=\left[0,\left[d_{\Omega}(X)\right]_{1,1}\right]
$$

if $N=1$. By using inequalities (67), $D_{\Omega}(X) \cap B_{r_{\max }}^{\infty}$ is a polytope. However, since

$$
\text { eva }: \mathbb{R}^{N} \rightarrow \mathbb{R}, \quad \operatorname{eva}(\vec{z})=\sum_{i=1}^{N} \pi e^{-2 h z_{i}} \theta_{v} z_{i}^{2}
$$


has no critical points in $D_{\Omega}(X) \cap B_{r_{\max }}^{\infty} \backslash\{\overrightarrow{0}\}$, the maximum of the extrinsic vertex area is attained in the boundary

$$
\operatorname{eva}(X, \Omega, h)=\max _{\vec{r} \in \partial\left(D_{\Omega}(X) \cap B_{r_{\max }}^{\infty}\right)}\left\{\sum_{i=1}^{N} \pi e^{-2 h r_{i}} \theta_{\nu} r_{i}^{2}\right\},
$$

and hence,

$$
\operatorname{evA}(X, \Omega, h)=\max _{Z \subset X}\left(\max _{\vec{r} \in \partial\left(D_{\Omega}(Z) \cap B_{r_{\max }}^{\infty}\right)}\left\{\sum_{i=1}^{N} \pi e^{-2 h r_{i}} \theta_{v} r_{i}^{2}\right\}\right),
$$

where $Z$ ranges on subsets of $X$. Moreover, if

$$
\left[d_{\Omega}(X)\right]_{i, j} \leq \frac{2-\sqrt{2}}{h}, \quad \forall i, \forall j
$$

the function eva is a convex function in the polytope $D_{\Omega}(X) \cap B_{r_{\max }}^{\infty}$, and hence its maximum is attained in the vertices of $D_{\Omega}(X) \cap B_{r_{\max }}^{\infty}$. Then, in the case $\left[d_{\Omega}(X)\right]_{i, j} \leq \frac{2-\sqrt{2}}{h}$ (minimal foams for instance), an estimation of the evA can be obtained using the vertex enumeration problem. In Sect. 5.5.2 we provide an algorithm using precisely the approach of the vertex enumeration problem.

Observe that since $Z$ is a subset of $X$, then the matrix of distances $\mathcal{D}_{\Omega}(Z)$ can be obtained from the matrix $\mathcal{D}_{\Omega}(X)$ by removing several rows and the corresponding columns.

Example 5.1 Let $\Omega$ be a domain inside a minimal foam $(h=0)$ such that the matrix of distances of the vertices $X=\{A, B, C\}$ of $\Omega$ is

$$
\mathcal{D}_{\Omega}(X)=\left(\begin{array}{llll}
0 & 1 & 2 & 4 \\
1 & 0 & 3 & 4 \\
2 & 3 & 0 & 4 \\
4 & 4 & 4 & 0
\end{array}\right)
$$

The following graph can be useful in order to represent the distances between vertices and the distances between vertices and the boundary $\partial \Omega$ :

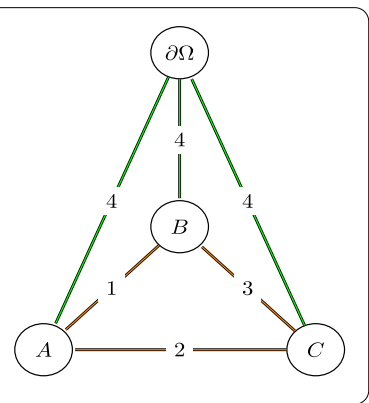


Figure 5 Domain $D_{\Omega}(X)$ from Example 5.1

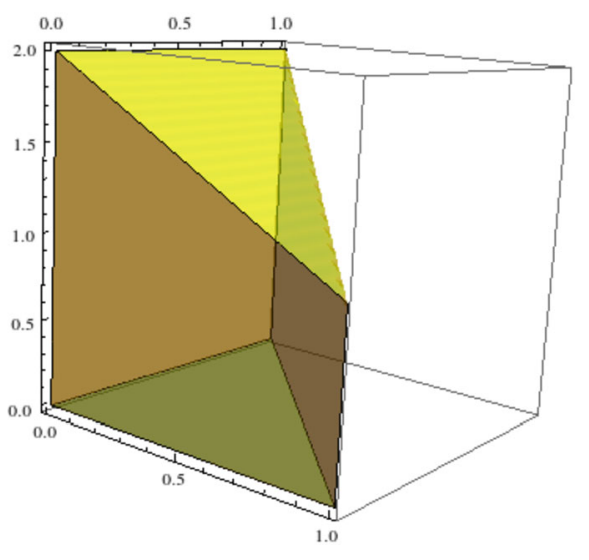

Then the reduced matrix of $X=\{A, B, C\}$ is just

$$
d_{\Omega}(X)=\left(\begin{array}{lll}
0 & 1 & 2 \\
1 & 0 & 3 \\
2 & 3 & 0
\end{array}\right)
$$

and the domain $D_{\Omega}(X) \cap B_{r_{\max }}^{\infty}$ is the domain bounded (see Fig. 5) by the following inequalities M.r $\leq b$ :

$$
\left\{\begin{array}{l}
r_{1}+r_{2} \leq 1 \\
r_{1}+r_{3} \leq 2 \\
r_{2}+r_{3} \leq 3 \\
-r_{1} \leq 0 \\
-r_{2} \leq 0 \\
-r_{3} \leq 0
\end{array}\right.
$$

where $M$ is a matrix of zeros and ones relating the different radii among themselves and with the boundary $\partial \Omega$ and $b$ is the vector of distances. The vertices of the polytope $D_{\Omega}(X) \cap B_{r_{\max }}^{\infty}$ are

$$
\{(0,0,0),(1,0,0),(0,1,0),(0,1,2),(0,1,1),(1,0,1)\}
$$

It is not hard to see that

$$
\operatorname{eva}(X, \Omega, 0)=\left(0^{2}+1^{2}+2^{2}\right) \theta_{v} \pi=5 \theta_{v} \pi
$$

If we choose $Z_{1}=\{B, C\}$, then

$$
\mathcal{D}_{\Omega}\left(Z_{1}\right)=\left(\begin{array}{lll}
0 & 3 & 4 \\
3 & 0 & 4 \\
4 & 4 & 0
\end{array}\right)
$$


with

$$
d_{\Omega}\left(Z_{1}\right)=\left(\begin{array}{ll}
0 & 3 \\
3 & 0
\end{array}\right)
$$

and eva $\left(Z_{1}, \Omega, 0\right)=9 \theta_{v} \pi$. But we can check that

$$
\operatorname{evA}(X, \Omega, 0)=\operatorname{eva}(\{A\}, \Omega, 0)=\operatorname{eva}(\{B\}, \Omega, 0)=\operatorname{eva}(\{C\}, \Omega, 0)=16 \theta_{v} \pi
$$

\subsubsection{Numerical solution of evA}

In the previous section we have explained the way to obtain an estimation of the evA from a set of points in a domain of the Euclidean space using the vertex enumeration problem. The algorithm can be summarized as Algorithm 1

Figure 6 shows the distribution of balls that gives the eva and the evA of a set of points. In this numerical simulation we have assumed that the set of vertices $X$ is a set of 8 points randomly contained in a ball of radius $R=10\left(\Omega=B_{10}(\overrightarrow{0})\right.$ and $\left.\partial \Omega=S_{10}(\overrightarrow{0})\right)$. Then, we have estimated the distance matrix $D_{\Omega}(X)$ from the set of inequalities $M . r \leq b$ as it appears in expression (68). To solve this system, the interior-point algorithm (see [16] for instance) has been used with the optimization tool in MATLAB. In Fig. 6(a), we show the maximum value of eva found to be $\operatorname{eva}(X, \Omega, 0)=29.0257 \theta_{v} \pi$ from the initial set of points.

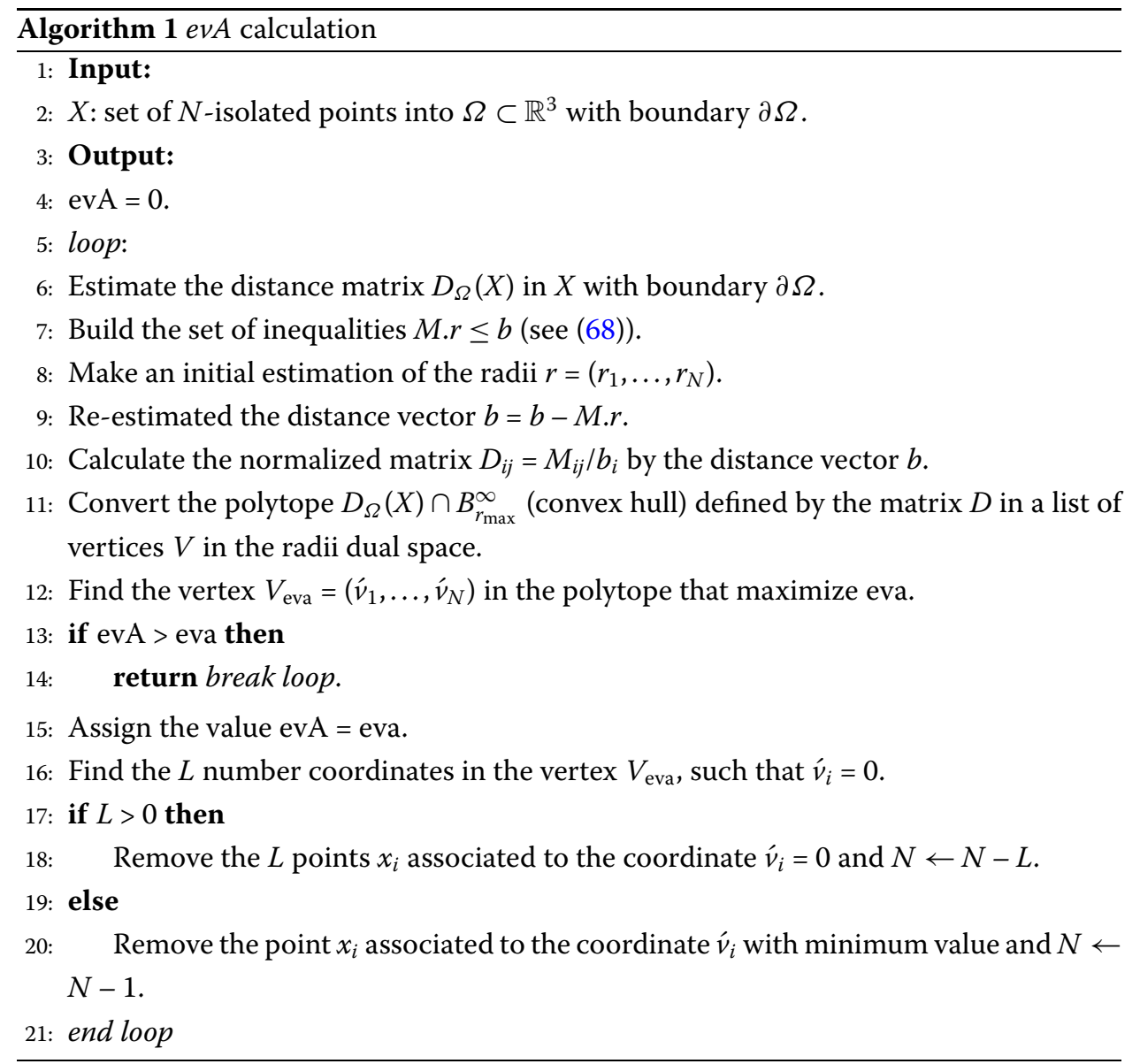




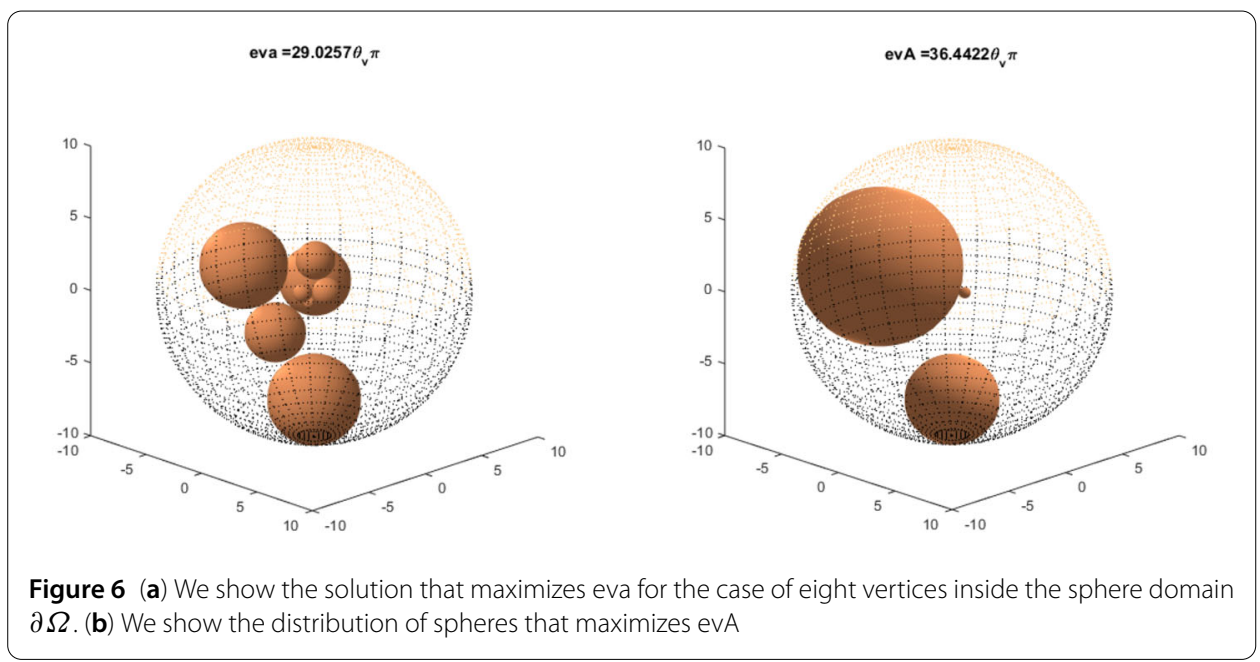

To compute evA, in this simulated example, we have used Algorithm 1. Namely, we have obtained a polytope $D_{\Omega}(X) \cap B_{r_{\max }}^{\infty}$ in the radii dual space corresponding to all the solutions that can be found from the set of inequalities. This polytope can be built from the algorithm proposed in [9] that converts a convex set of constraint inequalities into a set of vertices over the intersections of those inequalities (see lines 9-11 in Algorithm 1). From the set of vertices $V$ defined in the polytope, we choose one vertex $V_{\text {eva }}=\left(\hat{v}_{1}, \ldots, \hat{v}_{N}\right)$ that maximizes eva. Then, we search the coordinates in the vertex $V_{\text {eva }}$ with $\hat{v}_{i}=0$ or with minimum value in the coordinate. This means that, for this solution, the points $x_{i}$ in $\Omega \subset$ $\mathbb{R}^{3}$ have a small or vanishing radius, so they can be eliminated. In this example, only three balls centered at three of the initial vertices survive in the final solution $\operatorname{ev} \mathrm{A}(X, \Omega, 0)=$ $36.4422 \theta_{v} \pi$, while the points left are absorbed. In Fig. 6 (b) the final distribution of balls is shown.

\section{Acknowledgements}

We would like to thank Frank Morgan for reminding us about the paper [1], where such volume comparison formulas appear in a more general setting of varifolds with generalized mean curvatures.

\section{Funding}

This work has been partially supported by the Spanish Government Ministerio de Economía y Competitividad (MINECO-FEDER), grant MTM2017-84851-C2-2-P, Ministerio de Ciencia, Innovación y Universidades, grant RTI2018-098651-B-C54 and by Universitat Jaume I, grant UJI-B2018-35 and grant UJI-B2018-44.

\section{Availability of data and materials}

All data generated or analysed during this study are included in this article.

\section{Competing interests}

The authors declare that they have no competing interests.

\section{Authors' contributions}

All authors contributed equally and significantly in writing this article. All authors read and approved the final manuscript.

\section{Author details}

'Departament de Matemàtiques-IMAC, Universitat Jaume I, Castelló, Spain. ${ }^{2}$ DTU Compute, Technical University of Denmark, Lyngby, Denmark. ${ }^{3}$ Departament de Llenguatges i Sistemes Informátics-INIT, Universitat Jaume I, Castelló, Spain.

\section{Publisher's Note}

Springer Nature remains neutral with regard to jurisdictional claims in published maps and institutional affiliations. 


\section{References}

1. Allard, W.K.: On the first variation of a varifold. Ann. Math. 95, 417-491 (1972)

2. Aref, H., Vainchtein, D.L.: The equation of state of a foam. Phys. Fluids 12(1), 23-28 (2000)

3. Cheng, S.Y. Li, P., Yau, S.T.: Heat equations on minimal submanifolds and their applications. Am. J. Math. 106(5) 1033-1065 (1984)

4. Fortes, M.A.: The surface energy of finite clusters of soap bubbles. Phys. Fluids 13(12), 3542-3546 (2001)

5. Fortes, M.A.: The surface energy of strained foam clusters. Philos. Mag. Lett. 83(2), 143-148 (2003)

6. Fortes, M.A., Morgan, F., Fátima Vaz, M.: Pressures inside bubbles in planar clusters. Philos. Mag. Lett. 87(8), 561-565 (2007)

7. Hutchings, M., Morgan, F., Ritoré, M., Ros, A.: Proof of the double bubble conjecture. Ann. Math. 155, 459-489 (2002)

8. Karp, L., Pinsky, M.: Volume of a small extrinsic ball in a submanifold. Bull. Lond. Math. Soc. 21, 87-92 (1989)

9. Kleder, M.: CON2VERT-constraints to vertices.

http://www.mathworks.com/matlabcentral/fileexchange/7894-con2vert-constraints-to-vertices

10. Kusner, R.: The number of faces in a minimal foam. Proc. R. Soc. Lond. Ser. A 439(1907), 683-686 (1992)

11. Kusner, R., Sullivan, J.M.: Comparing the Weaire-Phelan equal-volume foam to Kelvin's foam. Forma 11(3), 233-242 (1996)

12. Lee, J.M.: Introduction to Smooth Manifolds, 2nd edn. Graduate Texts in Mathematics, vol. 218. Springer, New York (2013)

13. Markvorsen, S.: On the heat kernel comparison theorems for minimal submanifolds. Proc. Am. Math. Soc. 97(3), 479-482 (1986)

14. Markvorsen, S., Palmer, V.: The relative volume growth of minimal submanifolds. Arch. Math. 79(6), 507-514 (2002)

15. Morgan, F:: Geometric Measure Theory: A Beginner's Guide, 3rd edn. Academic Press, San Diego (2000)

16. Potra, F.A., Wright, S.J.: Interior-point methods. J. Comput. Appl. Math. 124, 281-302 (2000)

17. Ross, S.: Bubbles and foams-new general law. Ind. Eng. Chem. 61(10), 48-57 (1969)

18. Sakai, T.: Riemannian Geometry. Translations of Mathematical Monographs, vol. 149. Am. Math. Soc., Providence (1996). Translated from the 1992 Japanese original by the author

19. Taylor, J.: The structure of singularities in soap-bubble-like and soap-film-like minimal surfaces. Ann. Math. (2) 103(3), 489-539 (1976)

\section{Submit your manuscript to a SpringerOpen ${ }^{\circ}$ journal and benefit from:}

- Convenient online submission

- Rigorous peer review

- Open access: articles freely available online

- High visibility within the field

- Retaining the copyright to your article

Submit your next manuscript at $\boldsymbol{~ s p r i n g e r o p e n . c o m ~}$ 